\title{
A WiFi-based Positioning Parking Guidance System
}

\section{Lingli Mei ${ }^{\mathrm{a}}$, Mingxi Cheng ${ }^{\mathrm{b}}$}

International School, Beijing University of Posts and Telecommunications, Beijing 102209, China

as94994395@bupt.edu.cn, beric@bupt.edu.cn

Keywords: WiFi-based, RSSI positioning, parking guidance system

\begin{abstract}
With the development of economic and the growth of the city, the number of vehicles has also increased rapidly, parking problems continue testing the ability of the city's public services. To solve this problem, we hope to find a more accurate and more convenient program to find the car, so we propose a WiFi-based positioning parking guidance system.
\end{abstract}

\section{Introduction}

With the increasing comprehensive national strength of our country, the automobile has become a necessary tool for daily travel. In order to reduce the dependence on the parking lot, we propose a WiFi-based positioning parking guidance system. In this system, routers are installed around the parking lot. When we need to park car, the car is located by WiFi and the location of the car is recorded in the mobile App. When we need to retrieve the car, mobile phone is located by WiFi and App compares the two position to find an optimal route to the car.

\section{System Framework.}

In terms of parking space management, the parking situation is stored in the database in order to record the parking usage situation. We use the parking guidance system to detect whether vehicles park in the parking space. Ultrasonic detector is installed above each parking space, the detector collects the parking information of each parking space timely [1, 2]. Node controllers connected with the detectors will collect information of these detectors by polling mode, and feedback to the central controller after data are compress encoded. The central controller processes data and send the parking information to the LED. Then the data is stored in the database server, web servers send the parking information to the mobile terminals. So the terminals obtain the information of empty parking spaces.

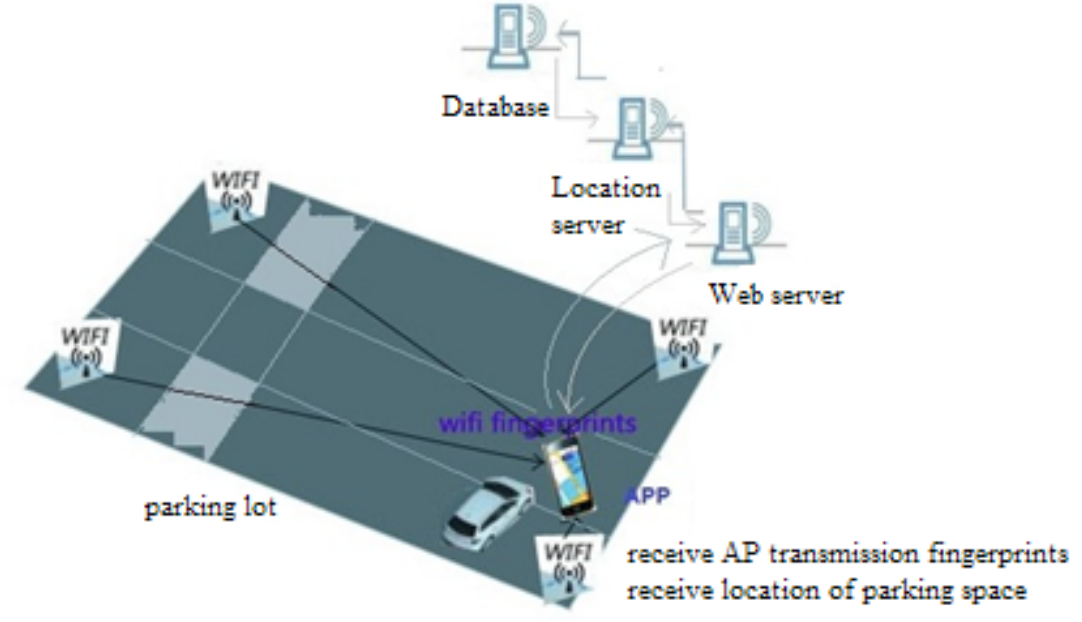

Fig. 1 System design

The WiFi-based indoor positioning system consist of two parts, the positioning server and the positioning client [3]. Software part mainly consist includes three aspects, web server, location server and App. Web server is used for external communication, it receives requests from outside and responds corresponding information. In the positioning stage, it will transfer data from the mobile terminal to the location server, and transmit position information obtained by the location server to the mobile terminal. In the guidance system, web server send the parking information obtained from 
database to terminal to connect the mobile terminal and location server. The positioning server is mainly used for the operation of the algorithm, it processes fingerprint vector data and match the results to the data in the database, then it send the location information obtained by the database to the web server. In the App, we can locate our parking space firstly and mark the position, when we need to retrieve the car, we locate the position of ourselves and get to the car through the guidance of the app. Moreover, the information of available parking space also send to the App through the guidance system. The framework of the WiFi-based positioning parking guidance system is shown in Figure 2.

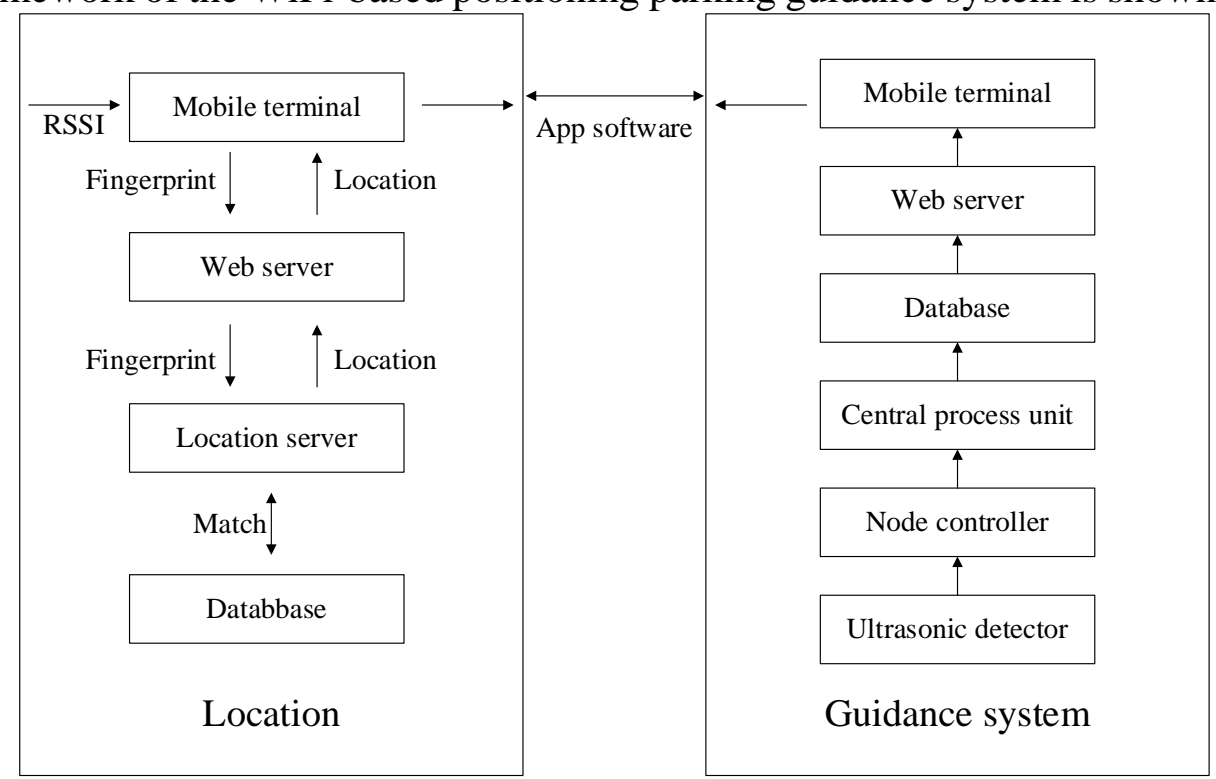

Fig. 2 System framework

\section{The Design of Position Fingerprint Database.}

The positioning algorithm mainly includes two stages: off-line training and on-line positioning. The task of the off-line training phase is to set up the one-one corresponding relationship between the intensity vector of the radio frequency signal and the position of the client to build a fingerprint database. In this stage, we use the way of experiment to measure the AP signal intensity of certain position, and choose the corresponding relationship between the mean value of several group data and the location, so as to build the fingerprint database $[4,5]$. The positioning stage is to use the signal intensity vector to match the fingerprint database, in order to obtain the target position estimation. The signal intensity vector is collected by the mobile terminal, and the information of the fingerprint information is corresponding to the database. Take Received Signal Strength Indication (RSSI) mean for each AP data collected, set a choice range $\left[R S S I-\sigma_{s} R S S I+\sigma\right]$ for each RSSI value of the AP, where $\sigma$ is empirical value of multiple experiments [6]. Find suitable position points in the fingerprint database meet the choice range, if there are $n$ position points to fall in this range, set the weight of these position points $1 / n$, and others 0 . After all AP do this procedure, choose the position of the maximum weight as the estimation position. If there are more than one position point with the same weight, compare signal intensity distance, take the smallest. Finally find the location through matching the contents of the fingerprint database.

\section{The Design of Client Module.}

The client is divided into 3 modules: Device module, Wlanscan module and Socket module. The communication between modules is shown in the Figure 3. 


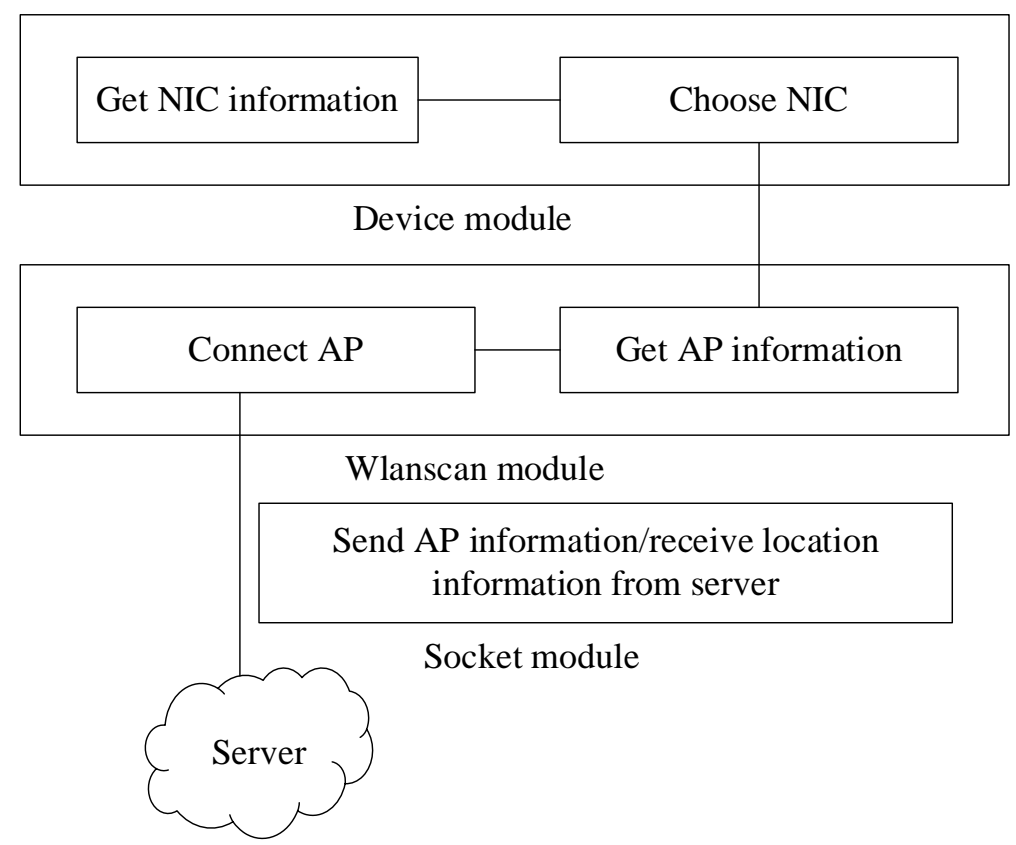

Fig. 3 The communication between modules

Wireless NIC selection function module: Call the basic API function to search for all NIC information on the machine, then list this information for users to choose. After user selects a wireless NIC, record the information of the NIC user selected.

Wireless NIC search function module: Search AP information around, and extract the information required by system to assemble. The running process of the module is as follows: Use the NIC information obtained from wireless NIC selection function module to open the wireless NIC, then it enables wireless card to transmit a frame in all available channels through functions, and receive the response information from the surrounding AP, finally select the information required by the system from the AP response message to assemble.

Information assemble module: Program matches and classifies the information according to the MAC address of AP, and then it will calculate mean value and standard deviation of RSSI values of information which have been classified. After calculating all the information, it will package the calculation value and the corresponding MAC address in the defined data structure.

Socket communication module: Used for data transmission between client and server. The module only has two procedure: connecting to the server and the data transmission. Single thread TCP communication need to define the IP address and port address of the server side in advance, after the execution of the above procedure, the client connects to the server successfully, the next step is data transmission.

System login module: This module realizes registration and verification login function, and is responsible for the page Jump.

Parking module: Simulate the parking spaces on the client interface, this interface module calls data transmitted between the other modules and the server to calculate the location, it also can show user's current position.

\section{The Design of Server Module.}

Login and log off: The design of Login interface is relatively simple, it only need users to enter the correct user name and password to log in, otherwise shows the warning of login failure, and reminds users to login once again. If users want to leave the system, users need to sign off the account to ensure security. When the user clicks the "sign off" button, the log off action is implemented.

User management: After the user successfully logged in, he will enter the welcome interface. In the navigation bar on the left, there are user management, device management, device location query, exit, and so on. By clicking on the function options, the corresponding functions are selected to perform the functions. The management of the user and the mobile terminal is mainly related to the 
increase of the user and the mobile terminal, delete, modify and query and so on, the function of these two parts is similar. User management mainly includes the registration of new users, the deletion of the existing users, user information changes and password changes, system administrator can realize the management of legal users of the system through this function. In mobile terminal management, when a new device is added to the system, the database adds a record correspondingly to record the information of the newly added device. When there is a device stop using for too old or other reasons, you can remove the device through deleting the device function, or makes the database of equipment information timely and accurately reflects the current situation of equipment by changing device information. When the user clicks the Add button, a div layer is popped up for users to fill in information. When the user completes filling it and clicks "OK" button, then it will perform insert operation to add a new user or a device. When a user wants to delete a record, he should select the item he want to delete, then click the delete button to perform the delete operation; if user clicks the delete button without choosing any item, it will give the "Please choose the item you want to delete." warning. After the user selects the item to be changed and clicks the update button, a div layer is popped up for users to fill in the change information, then user should click the OK button to perform the update operation.

\section{Conclusion}

Wireless location technology has received more and more attention and it is widely applied in all walks of life. However, when it comes to Indoor Location, GPS location has relatively low accuracy or even unable to locate. Combined with the phenomenon of contemporary parking difficult, we propose a WiFi-based positioning parking guidance system which can be realized.

\section{References}

[1] Jian Zhu, Hai Zhao, Peigang Sun. Equilateral Triangle Localization Algorithm Based On Rssi Mean Value. Journal of Northeastern University. 2007: 2-4.

[2] Wenzhou Chen. The Research and Application of WiFi Technology. Journal of Data Communication. 2008(2).

[3] Lashkari A H, Parhizkar B, Ngan M N A. WIFI-based indoor positioning system[C]//Computer and Network Technology (ICCNT), 2010 Second International Conference on. IEEE, 2010: 76-78.

[4] Zhan J, Liu H L, Liu S G, et al. The study of dynamic degree weighted centroid localization algorithm based on RSSI[J]. Dianzi Xuebao(Acta Electronica Sinica), 2011, 39(1): 82-88.

[5] Park D W, Park J G. An enhanced ranging scheme using WiFi RSSI measurements for ubiquitous location[C]//Computers, Networks, Systems and Industrial Engineering (CNSI), 2011 First ACIS/JNU International Conference on. IEEE, 2011: 296-301.

[6] Liu H H, Yang Y N. WiFi-based indoor positioning for multi-floor environment[C]//TENCON 2011-2011 IEEE Region 10 Conference. IEEE, 2011: 597-601. 